\title{
What clinical features are most useful to distinguish definite multiple system atrophy from Parkinson's disease?
}

\author{
G K Wenning, Y Ben-Shlomo, A Hughes, S E Daniel, A Lees, N P Quinn
}

Department of Neurology, University Hospital, Innsbruck, Austria

G K Wenning

Department of Social Medicine, University of Bristol, Bristol, UK Y Ben-Shlomo

Austin and Repatriation Medical Centre, Heidelberg

West, Victoria,

Australia

A Hughes

Parkinson's Disease Society Research

Centre, London, UK

$S$ E Daniel

A Lees

Institute of Neurology, Queen Square,

London, UK

N P Quinn

Correspondence to: Professor N Quinn, University Department of Clinical Neurology, Institute of Neurology, Queen Square, London WC1N 3BG, UK

Received 20 April 1999 and in revised form

27 October 1999

Accepted 3 December 1999

\begin{abstract}
Objectives-Few studies have attempted to identify what premortem features best differentiate multiple system atrophy (MSA) from Parkinson's disease (PD). These studies are limited by small sample size, clinical heterogeneity, or lack of postmortem validation. We evaluated the sensitivity and specificity of different clinical features in distinguishing pathologically established MSA from PD.

Methods-One hundred consecutive cases of pathologically confirmed PD and 38 cases of pathologically confirmed MSA in one Parkinson's disease brain bank were included. All cases had their clinical notes reviewed by one observer (AH). Clinical features were divided into two groups: those occurring up to 5 years after onset of disease and those occurring up to death. Statistical analysis comprised multivariate logistic regression analysis to choose and weight key variables for the optimum predictive model.
\end{abstract}

Results-The selected early features and their weightings were: autonomic features (2), poor initial levodopa response (2), early motor fluctuations (2), and initial rigidity (2). A cut off of 4 or more on the ROC curve resulted in a sensitivity of $87.1 \%$ and specificity of $70.5 \%$. A better predictive model occurred if the following features up to death were included: poor response to levodopa (2), autonomic features (2), speech or bulbar dysfunction (3), absence of dementia (2), absence of levodopa induced confusion (4), and falls (4). The resulting ROC curve based on individual scores showed a best cut off score of at least 11 of 17 (sensitivity $90.3 \%$, specificity $\mathbf{9 2 . 6 \%}$ ).

Conclusions-Predictive models may help differentiate MSA and PD premortem. Hitherto poorly recognised features, suggestive of MSA, included preserved cognitive function and absence of psychiatric effects from antiparkinsonian medication. Diagnostic accuracy was higher in those models taking into account all clinical features occurring up to death. Further studies need to be based on new incident cohorts of parkinsonian patients with subsequent neuropathological evaluation. (F Neurol Neurosurg Psychiatry 2000;68:434-440)

Keywords: multiple system atrophy; Parkinson's disease; clinicopathological study; differential diagnosis
Multiple system atrophy (MSA) is a clinically and pathologically distinctive neurodegenerative disease. ${ }^{1}$ Historically, it has been described in three different ways: olivopontocerebellar atrophy (OPCA), ${ }^{2}$ striatonigral degeneration (SND), ${ }^{3}$ and the Shy-Drager syndrome, (SDS). ${ }^{4}$ The considerable clinicopathological overlap among these three subgroups led Graham and Oppenheimer to introduce MSA as an umbrella term. ${ }^{5}$ There is now general agreement that MSA is one distinct entity among many multisystem degenerations. In recent years glial cytoplasmic inclusions have been described in oligodendrocytes that are present in the brains of all patients with MSA of whatever denomination..$^{6-8}$ This abundant presence of glial cytoplasmic inclusions in all clinical subtypes of MSA has introduced further grounds for considering SDS, SND, and many cases of sporadic OPCA together as MSA.

It is often difficult to distinguish clinically between MSA and Parkinson's disease, particularly early in the disease. In the United Kingdom Parkinson's Disease Society Research Centre (UKPDSRC) study of 35 cases of MSA, one third of the patients died with a misdiagnosis. Even when a correct premortem diagnosis of MSA had been made, this still took an average of 4 years after presentation. ${ }^{9}$ Poor diagnostic sensitivity for MSA among movement disorder specialists has been reported by Litvan et al..$^{10}$ Mean rater sensitivity in their study was $56 \%$ at the first clinic visit and this had increased to $69 \%$ by the last visit. However, primary neurologists, who followed up the patients clinically, achieved even lower sensitivities of only $25 \%$ and $50 \%$ at the first and last visit respectively. The reasons for such poor diagnostic accuracy have not been studied systematically. However, overlapping clinical features probably contribute. ${ }^{11-12}$ For example, asymmetry of the cardinal features of parkinsonism normally associated with PD is also seen in most patients with MSA. ${ }^{13}$ In addition, atypical features that suggest non-idiopathic disease such as poor response to levodopa, autonomic failure, cerebellar incoordination, and pyramidal signs as well as severe immobility may all take several years to become evident. However, as prognosis and treatment response is different for patients with MSA compared with $\mathrm{PD}^{14}{ }^{15}$ a correct diagnosis is important. We are aware of only three previous studies which have attempted to identify what premortem features best differentiate MSA from PD. ${ }^{10}{ }^{16}$ All three have had 20 or fewer cases 
of MSA and have compared these either to other parkinsonian patients ${ }^{10}$ or to various other neurological conditions. ${ }^{10} 17$ The study by Albanese et al provides the most realistic setting as it evaluated consecutive parkinsonian outpatients. ${ }^{16}$ However, features were compared with a clinical diagnosis, partially based on those features, without pathological confirmation, therefore resulting in an inherent circularity. In the present study we aimed to determine the sensitivity and specificity of different features in distinguishing pathologically established MSA from PD. We also determined the best combination of predictor variables using multivariate methods in an attempt to produce a fairly simple clinical diagnostic index.

\section{Methods}

SELECTION OF SUBJECTS

We studied 100 consecutive patients with pathologically confirmed PD and 38 patients with pathologically confirmed MSA, whose brains were collected by the Parkinson's Disease Society Brain Bank (PDSBB) in London, UK, between June 1987 and July 1993. Most of the patients with PD and some of the patients with MSA were registered donors and many had been prospectively assessed annually by neurologists associated with the PDSBB. About a third of the patients with MSA, all with a clinical diagnosis of MSA, had been seen in life by one of us (NPQ). Selected clinicopathological features of some of the patients with PD or MSA have been previously reported. ${ }^{9}{ }^{11} 17-22$

\section{POSTMORTEM DIAGNOSIS}

The defining histological characteristics for PD were depletion of pigmented neurons in the substantia nigra and locus ceruleus, with Lewy bodies in some remaining nerve cells and elsewhere in the nervous system. The striatum was normal and there were no glial cytoplasmic inclusions or additional pathology to account for the presence of parkinsonism. ${ }^{18}$

All cases of MSA satisfied the neuropathological criteria proposed by Gray et $a l^{23}$ and modified by Quinn ${ }^{24}$ : neuronal cell loss and gliosis in a selection (at least two) of the following structures: striatum, substantia nigra, locus ceruleus, pontine nuclei and middle cerebellar peduncles, cerebellar Purkinje cells, inferior olives, and dorsal vagal nuclei; in addition, the presence of characteristic glial cytoplasmic argentophilic inclusions in the primary or secondary motor areas and basal ganglia. Because the striatal MSA lesion may seem subtle or even absent using conventional histological methods ${ }^{25}$ immmunohistochemistry for glial fibrillary acidic protein (GFAP) was performed in all patients in addition to routine stains such as cresyl violet (Nissl) and Luxol fast blue. ${ }^{26}$

CLINICAL VARIABLES

All clinical notes were reviewed by one observer (AH) who classified all subjects to maintain standardisation of coding. The relatively large number of variables were initially grouped into logical subheadings to enable a more rational approach to data analysis. These were as follows: (a) sociodemographic, (b) clinical, (c) drug side effects, and (d) miscellaneous features. In some cases, the same variable was classified twice, either at onset or early in the course of the disease, or alternatively at any time in the clinical history. This distinction is useful in determining which features may be early predictors.

Autonomic failure was defined as the presence of any of the following: symptomatic postural hypotension, urinary urge incontinence, faecal incontinence, urinary retention requiring catheterisation, or persistent erectile failure.

Akinesia (usually with rigidity) was necessary for a diagnosis of parkinsonism. However, tremor was neither obligatory nor sufficient on its own. Response to levodopa, based on the estimate in the records of either the patient or physician, or both, was graded on a four point scale $(1=0 \%-29 \%, 2=30 \%-49 \%$ (both "poor"), $3=50 \%-69 \%$ ("good"), and 4=70-100\% ("excellent”)). Subsequently, for the purpose of analysis, levodopa response was defined as poor (0\%-49\%) or definite (50\%-100\%). Early fluctuations, dyskinesias, neuropsychiatric toxicity, and dementia were defined as occurring within 5 years of onset. Cerebellar features recorded included limb ataxia, intention tremor, gait ataxia, and nystagmus, in isolation or in combination. Pyramidal features comprised equivocal or extensor plantar responses and hyperreflexia. Speech or bulbar features comprised dysarthria, dysphagia, and excessive sialorrhoea. "Other signs" is a catch all heterogeneous category that included any other features seen that were atypical for PD.

\section{STATISTICAL METHODS}

For each variable, the sensitivity, specificity, and positive and negative predictive values were calculated as well as the $\chi^{2}$ value. The diagnostic utility of each variable to correctly detect MSA is for a combination of pathologically established cases of MSA and PD only. The performance of each variable was summarised using the Youden index (sensitivity plus specificity-100). This measure gives equal weight to both sensitivity and specificity. Because of the collinearity between certain clinical variables, it was decided not to enter all variables into a stepwise model but rather, within each subgroup, to determine the best predictor or combinations of predictors using multivariate logistic regression analysis with the postmortem diagnosis (MSA or PD) as the outcome variable. The best predictor(s) from each subgroup were then entered into a new logistic model to produce the most parsimonious model. Those variables that remained significant were then weighted by their $\beta$ coefficient, and these weightings were then used to produce a simplified clinical score. The exact values were not used, as the intention was to enable clinicians in a normal setting to calculate a score without the use of calculators. For each case, the score was calculated and an ROC curve was produced from which we subjectively chose the most sensible cut off for clinical practice. Because of missing data, the analyses were not always based on the total 
Table 1 Diagnostic and predictive value of various clinical features compared with postmortem diagnosis of multiple system atrophy and Parkinson's disease

\begin{tabular}{|c|c|c|c|c|c|c|c|c|c|}
\hline Variables & & $M S A$ & $P D$ & $\begin{array}{l}X^{2} \text { value } \\
\text { on } 1 d f\end{array}$ & $\begin{array}{l}\text { Sensitivity } \\
(\%)\end{array}$ & $\begin{array}{l}\text { Specificity } \\
(\%)\end{array}$ & $P P V$ & $N P V$ & $\begin{array}{l}\text { Youden } \\
\text { index }\end{array}$ \\
\hline \multicolumn{10}{|l|}{ Sociodemographic: } \\
\hline \multirow[t]{2}{*}{ Sex } & $(\mathrm{M})$ & 20 & 64 & 0.22 & 52.6 & 36.0 & 23.8 & 66.7 & -11.4 \\
\hline & $(\mathrm{F})$ & 18 & 36 & & & & & & \\
\hline \multirow[t]{2}{*}{ Age onset $\leqslant 55 y$} & (Y) & 16 & 20 & 0.008 & 42.1 & 80.0 & 44.4 & 78.4 & 22.1 \\
\hline & $(\mathrm{N})$ & 22 & 80 & & & & & & \\
\hline \multicolumn{10}{|l|}{ Initial features: } \\
\hline \multirow[t]{2}{*}{ Symmetric onset } & $(\mathrm{Y})$ & 9 & 28 & 0.99 & 28.1 & 71.7 & 24.3 & 75.5 & -0.2 \\
\hline & $(\mathrm{N})$ & 23 & 71 & & & & & & \\
\hline \multirow[t]{2}{*}{ Initial $\mathrm{HY}>2$} & $(\mathrm{Y})$ & 13 & 21 & 0.002 & 54.2 & 78.1 & 38.2 & 87.2 & 32.3 \\
\hline & $(\mathrm{N})$ & 11 & 75 & & & & & & \\
\hline \multirow[t]{2}{*}{ Initial rigidity } & $(\mathrm{Y})$ & 21 & 30 & 0.005 & 56.8 & 69.7 & 41.2 & 81.2 & 26.5 \\
\hline & $(\mathrm{N})$ & 16 & 69 & & & & & & \\
\hline \multirow[t]{2}{*}{ Initial poor levodopa response } & (Y) & 18 & 22 & 0.0001 & 58.1 & 76.8 & 45.0 & 84.9 & 34.9 \\
\hline & $(\mathrm{N})$ & 13 & 73 & & & & & & \\
\hline \multicolumn{10}{|l|}{ Clinical features ${ }^{\star}$ : } \\
\hline \multirow[t]{2}{*}{ Tremor } & $(\mathrm{Y})$ & 23 & 76 & 0.07 & 60.5 & 24.0 & 23.2 & 61.5 & -15.5 \\
\hline & $(\mathrm{N})$ & 15 & 24 & & & & & & \\
\hline \multirow[t]{2}{*}{ Rigidity } & $(\mathrm{Y})$ & 37 & 96 & 0.7 & 97.4 & 4.0 & 27.8 & 80.0 & 1.4 \\
\hline & $(\mathrm{N})$ & 1 & 4 & & & & & & \\
\hline \multirow[t]{2}{*}{ Bradykinesia } & $(\mathrm{Y})$ & 36 & 90 & 0.16 & 97.3 & 10.0 & 28.6 & 90.9 & 7.3 \\
\hline & $(\mathrm{N})$ & 1 & 10 & & & & & & \\
\hline \multirow{2}{*}{ Autonomic } & (Y) & 32 & 26 & 0.0001 & 84.2 & 73.7 & 55.2 & 92.4 & 57.9 \\
\hline & $(\mathrm{N})$ & 6 & 73 & & & & & & \\
\hline \multirow{2}{*}{ Cerebellar } & (Y) & 12 & 1 & 0.0001 & 32.4 & 99.0 & 92.3 & 79.7 & 31.4 \\
\hline & $(\mathrm{N})$ & 25 & 98 & & & & & & \\
\hline \multirow[t]{2}{*}{ Pyramidal } & (Y) & 18 & 7 & 0.0001 & 50.0 & 92.9 & 72.0 & 83.6 & 42.9 \\
\hline & $(\mathrm{N})$ & 18 & 92 & & & & & & \\
\hline Speech and/or bulbar & (Y) & 33 & 21 & 0.0001 & 86.8 & 78.8 & 61.1 & 94.0 & 65.6 \\
\hline & $(\mathrm{N})$ & 5 & 78 & & & & & & \\
\hline Dementia absent & (Y) & 29 & 44 & 0.0001 & 82.9 & 54.6 & 39.7 & 89.8 & 37.5 \\
\hline & $(\mathrm{N})$ & 6 & 53 & & & & & & \\
\hline Early dementia absent & (Y) & 29 & 78 & 0.01 & 100.0 & 17.9 & 27.1 & 100.0 & 17.9 \\
\hline & $(\mathrm{N})$ & 0 & 17 & & & & & & \\
\hline Asymmetric disease & (Y) & 13 & 54 & 0.033 & 34.2 & 45.5 & 19.4 & 64.3 & -20.3 \\
\hline & $(\mathrm{N})$ & 25 & 45 & & & & & & \\
\hline Drug side effects: & & & & & & & & & \\
\hline Toxic confusion absent & $(\mathrm{Y})$ & 27 & 27 & 0.0001 & 84.4 & 71.9 & 50.0 & 93.2 & 56.3 \\
\hline & $(\mathrm{N})$ & 5 & 69 & & & & & & \\
\hline Early toxic confusion absent & (Y) & 27 & 80 & 0.04 & 100.0 & 14.0 & 25.2 & 100.0 & 14.0 \\
\hline & $(\mathrm{N})$ & 0 & 13 & & & & & & \\
\hline Fluctuations & $(\mathrm{Y})$ & 15 & 58 & 0.18 & 46.9 & 39.6 & 20.5 & 69.1 & -13.5 \\
\hline & $(\mathrm{N})$ & 17 & 38 & & & & & & \\
\hline Early fluctuations & (Y) & 14 & 19 & 0.006 & 45.2 & 80.0 & 42.4 & 81.7 & 25.2 \\
\hline & $(\mathrm{N})$ & 17 & 76 & & & & & & \\
\hline Dyskinesia & $(\mathrm{Y})$ & 15 & 59 & 0.14 & 45.5 & 39.8 & 20.3 & 68.4 & -14.7 \\
\hline & $(\mathrm{N})$ & 18 & 39 & & & & & & \\
\hline Early dyskinesias & $(\mathrm{Y})$ & 8 & 18 & 0.43 & 25.0 & 81.4 & 30.8 & 76.7 & 6.4 \\
\hline & $(\mathrm{N})$ & 24 & 79 & & & & & & \\
\hline Other features: & & & & & & & & & \\
\hline Other signs & $(\mathrm{Y})$ & 30 & 12 & 0.0001 & 78.9 & 88.0 & 71.4 & 91.7 & 66.9 \\
\hline Other causes & (Y) & 3 & 20 & 0.09 & 7.9 & 80.0 & 13.0 & 69.6 & -12.1 \\
\hline & $(\mathrm{N})$ & 35 & 80 & & & & & & \\
\hline Depression & $(\mathrm{Y})$ & 14 & 56 & 0.24 & 51.9 & 35.6 & 20.0 & 70.5 & -12.5 \\
\hline & $(\mathrm{N})$ & 13 & 31 & & & & & & \\
\hline Falls & (Y) & 31 & 54 & 0.001 & 86.1 & 44.3 & 36.5 & 89.6 & 30.4 \\
\hline & $(\mathrm{N})$ & 5 & 43 & & & & & & \\
\hline Family history & $(\mathrm{Y})$ & 2 & 4 & 0.65 & 5.9 & 96.0 & 33.3 & 75.0 & 1.9 \\
\hline & $(\mathrm{N})$ & 32 & 96 & & & & & & \\
\hline
\end{tabular}

PPV=positive predictive value; NPV=negative predictive value.

${ }^{\star}$ Recorded at any time up to death.

Youden index $=$ sensitivity plus specificity minus 100 . Values can vary from between 100 to-100, a larger value indicating a more useful feature.

sample size. Where a clinical feature was not mentioned we assumed that the absence of its recording in the notes indicated that it was not present. However, we cannot be certain that the relevant clinician actually looked for this feature and thus its absence may simply indicate a failure to see or record it. For certain variables, such as initial Hoehn and Yahr score or levodopa response, we have omitted all observations with missing data from the multivariate analysis and have not attempted to impute results.

Results

Data were available on 38 patients with MSA and 100 patients with PD. The mean age of onset for patients with MSA was 55.9 years
(SD 10.6) compared with 62.4 years (SD 9.3) for PD $(p<0.001)$. Mean disease duration until death was also significantly shorter for the MSA than the PD group (6.8 years $v 13.2$ years, $p<0.0005)$. The initial Hoehn and Yahr score was significantly worse for patients with MSA compared with those with PD $(2.4 v 1.9$, $\mathrm{p}<0.01)$. The percentage of patients with age at onset less than 55 years was twice as high in the MSA than in the PD group $(\mathrm{p}<0.01)$. At the initial neurological examination, asymmetry was present in $72 \%$ of both patients with PD and those with MSA. A poor initial levodopa response was more common in the MSA group (58\%), but it also occurred in some $(23 \%)$ patients with PD $(p<0.0005)$. Initial rigidity was seen more often among the 
MSA (57\%) than the PD (30\%) group $(\mathrm{p}<0.005)$.

The diagnostic value of each clinical feature by itself is shown in table 1 . The following were the best predictors, in order of the Youden index, and were chosen to enter into the multivariate models: age of onset $\leqslant 55$ years, atremulous or akinetic at onset, asymmetric disease, initial Hoehn and Yahr stage 3 or greater, poor initial levodopa response, early fluctuations, absence of toxic (induced by dopaminomimetic and/or anticholinergic drugs) confusion or hallucination, speech or bulbar features, autonomic features, pyramidal features, cerebellar features, falls, absence of dementia, and other clinical signs. Most of the standard clinical features that are used in the diagnosis of MSA appear in the above list. However, some of these-for example, cerebellar features-are very useful when present (high specificity) but have limited sensitivity as they are only found in a smaller subgroup of patients than autonomic features. Their predictive value is therefore limited, and not all the standard diagnostic features remained in the final multivariate model.

The final selected logistic regression model, coefficients, and weightings are shown in table 2 , where pathologically established cases of MSA were coded as 1 and PD cases as 0 . If a patient had any of these specific features they were given the following score (otherwise they scored zero): poor initial response to levodopa (2), autonomic features present (2), speech or bulbar problems present (3), absence of dementia (2), absence of toxic confusion (4), falls present (4). A patient could therefore score between 0 and 17 . By calculating an individual patient's score, it was possible to plot an ROC curve of sensitivity against 1-specificity (fig 1). We did this both using the exact coefficients and the rounded coefficients from table 2. As the results were almost identical we have only presented the rounded coefficients. The best compromise score is 11 or more out of 17 , which results in a sensitivity of $90.3 \%$ and specificity of $92.6 \%$.

It is clear that individual patient's scores can vary over time as they develop further symptoms and signs. Furthermore, these data are based on patients followed up until death and thus any features have had the maximal time to emerge. As such, the model is rather limited clinically as it is desirable to diagnose patients earlier than death. Absence of a feature at any one time does not mean it will not emerge in the future. Unfortunately the time from symptom onset to the development of autonomic, cerebellar, or pyramidal features was not

Table 2 Association between pathological diagnosis of MSA or PD using multivariate regression modelling

\begin{tabular}{lllll}
\hline Clinical features $^{*}$ & $\beta$-Coefficient & $S E$ & p Value & Weighting \\
\hline Initial poor levodopa response & 2.4 & 0.95 & 0.01 & 2 \\
Autonomic features & 2.4 & 0.91 & 0.007 & 2 \\
Speech and/or bulbar features & 3.1 & 1.0 & 0.002 & 3 \\
Absence of dementia & 2.2 & 0.96 & 0.02 & 2 \\
Absence of toxic confusion & 3.7 & 1.1 & 0.001 & 4 \\
Falls & 3.7 & 1.4 & 0.01 & 4 \\
\hline
\end{tabular}

$\star$ Recorded at any time up to death.

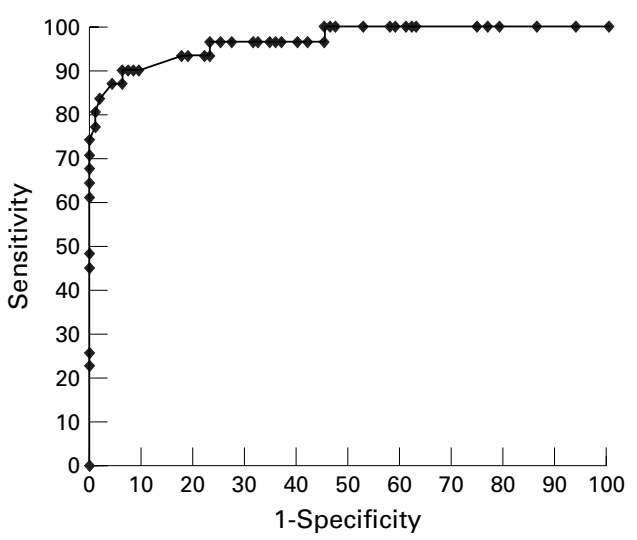

Figure 1 ROC curve of sensitivity plotted against 1 -specificity using the clinical index score $(0-17)$ to distinguish MSA from PD and including clinical features which may have developed at any time from disease onset to death using exact coefficients.

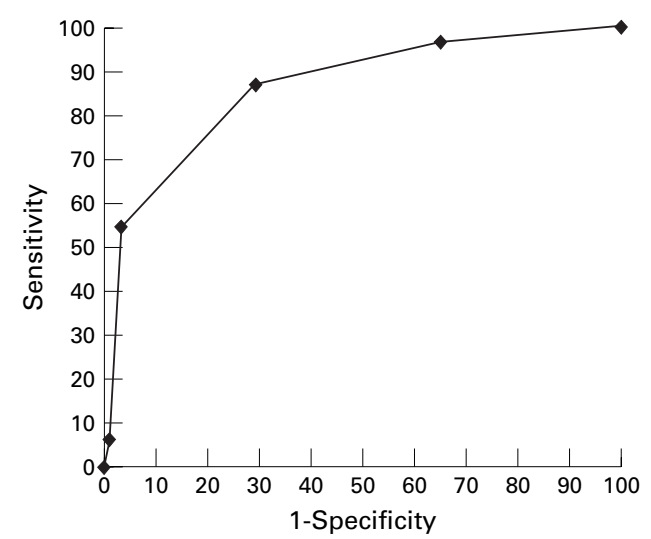

Figure 2 ROC curve of sensitivity plotted against 1 -specificity using the modified clinical index score (0-8) to distinguish MSA from PD and including clinical features present from onset, after initial drug treatment, or within the first 5 years of disease progression.

recorded. We therefore repeated the modelling using the features that were recorded at presentation, after initial treatment, or within the first 5 years after onset of symptoms. We also included autonomic features as these have been shown to be almost always present in cases of MSA and are a very common presenting feature, therefore being present relatively early in the natural history of the disease. This repeat analysis identified four variables as significant predictors with the following weightings; autonomic features present (2), poor initial response to levodopa (2), early fluctuations (2), initial rigidity (2). A patient could therefore score between 0 and 8 . The ROC curve for this initial clinical score is shown in figure 2 . In this case, a cut off of $\geqslant 4$ results in a sensitivity of $87.1 \%$ and specificity of $70.5 \%$.

\section{Discussion}

Before considering the relevance of our findings we need to draw attention to the drawbacks of our study:

(1) We analysed clinical data that were based on a combination of retrospective extraction of hospital notes and, when available, annual prospective UKPDS BB report forms. Ideally, an incident cohort of parkinsonian patients should 
be established with standardised high quality data on symptoms and examination and neuropathological confirmation. However, no such cohort currently exists so current research is limited to clinicopathological series of patients.

(2) The sample of subjects we have analysed was collected by a brain bank specifically interested in parkinsonian disorders and is therefore likely to overrepresent atypical or complex cases. It is not known therefore if the features seen are representative of all patients with MSA or PD. The utility of some features, such as response to levodopa, which may have a better predictive value in a more typical community sample, may have been underestimated.

(3) Despite the relatively large sample of cases of MSA, this analysis is still only based on limited numbers and is therefore prone to random error. Further work should try to replicate and validate our results in a different independent sample of patients.

(4) We simplified the diagnostic problem by limiting our comparison to patients with MSA or PD, thereby excluding other causes of parkinsonism, particularly progressive supranuclear palsy (PSP) which may symptomatically overlap with both.

Despite these inevitable shortcomings, our study suggests that the following clinical features may help in the differential diagnosis of MSA versus PD: poor initial response to levodopa and absent psychiatric side effects from antiparkinsonian drugs; dysarthria and dysphagia; recurrent falls; autonomic failure; and preserved cognitive function on bedside testing. These features are similar to those independently determined in another clinicopathological study, although levodopa responsiveness and bulbar symptoms were not specifically mentioned. ${ }^{10}$ However, that study chose to overrepresent many rare causes of neurological disease, so that cases of PD only comprised $17 \%$ of the non-MSA group, a rather unrealistic situation. This means that the utility of certain clinical features is distorted by the balance of other conditions that may mimic MSA or PD.

Both that study and ours identified preserved cognitive function as a useful clinical feature hitherto poorly recognised among neurologists to differentiate MSA from PD (see later). Our multivariate analysis enabled us to derive a clinical score out of 17 which permitted good discrimination between groups. For example, a cut off of 11 or more had a sensitivity of $90.3 \%$ and a specificity of $92.6 \%$. However, this model is based on a complete follow up of patients until death. Its utility in early disease is limited as absence of a symptom or sign at this stage does not mean that it will not develop later on. In this respect it is interesting to note that our second model, although weaker, still had reasonable sensitivity and specificity. Clinical suspicion should arise in patients, early in their natural history, who score more than 4 out of 8 on this simple score, and diligent follow up is necessary to detect further atypical features.
The following second visit features failed to differentiate between the groups: symmetric onset, akinesia, tremor, levodopa induced motor complications, and depression, indicating considerable overlap between PD and MSA. This suggests that except for producing levodopa unresponsiveness the striatal degeneration that is almost invariably present in MSA $^{921}$ does not significantly modify the nature of parkinsonism (as recorded in the case notes) that is caused by the nigral pathology alone in PD. Therefore, based on the present data it seems unwise to diagnose MSA by analysing features of parkinsonism alone (without knowledge of levodopa response).

Those clinical features that differentiated PD and MSA over the long term course of the disease will now be discussed in more detail.

INITIAL RESPONSE TO LEVODOPA

It is widely accepted that an excellent response to levodopa is expected in patients with PD. In fact, definite and sustained levodopa benefit is part of the UK PDS BB diagnostic criteria for PD. $^{20}$ Surprisingly, $23 \%$ of our patients with PD showed a poor initial response to levodopa compared with $58 \%$ poor initial responders within the MSA group. There may be several reasons why patients with PD fail to respond to levodopa. Some patients with PD who are functioning at a high level at initial diagnosis may not have an impressive response to levodopa because of a ceiling effect. Similarly, clinicians may not push levodopa to adequate doses. However, some pathologically established cases of PD with no appreciable response to levodopa have been reported. ${ }^{27}$ Overall, patients with PD were more likely to show fluctuations than the patients with MSA, yet when these did occur in MSA, they almost always (93\%) were noted within 5 years of onset. As early fluctuations more commonly occur in young patients with $\mathrm{PD}^{28}$ it is possible that the difference may reflect an age effect as patients with MSA were significantly younger than those with PD.

COGNITIVE STATUS AND PSYCHIATRIC SIDE EFFECTS TO ANTIPARKINSONIAN MEDICATION Our data suggest that dementia is common in advanced PD, but infrequent in MSA and early $\mathrm{PD}$, in line with previous literature reports. The absence of dementia therefore fails to distinguish MSA from PD early in the disease process. Most recent estimates of the prevalence of dementia in clinically diagnosed PD are in the order of $20 \%$, but the cumulative risk is about $40 \%{ }^{29}$ Visual hallucinations and other psychotic phenomena may be induced by antiparkinsonian drugs in otherwise uncomplicated PD, but can also occur in the absence of drug therapy in patients with dementia with Lewy bodies. ${ }^{30}$ By contrast, patients with MSA rarely develop dementia or psychiatric side effects from their medication, ${ }^{22}$ perhaps because the structural (particularly glial) change is largely limited to the brainstem and basal ganglia and related motor systems, ${ }^{6-9}$ and also because they tend to be younger. In addition, once a patient has been identified as having MSA many 
clinicians will stop or reduce levodopa or avoid increasing it to high dosages. Cholinergic deficits are less severe in MSA than in PD, ${ }^{31}$ although definitive neuropathological studies of the nucleus basalis of Meynert in MSA brains have not been reported.

AXIAL FUNCTION: SPEECH, SWALLOWING, AND EQUILIBRIUM

Speech impairment develops in virtually all patients with MSA, in whom it tends to be dominated by hypophonic monotony or a scanning quality according to the parkinsonian or cerebellar subtype. ${ }^{32}$ Dysarthria tends to develop earlier, be more common, more severe, and be associated with more marked dysphagia and sialorrhoea in MSA compared with PD.

Impaired postural reflexes with resultant falls cause major disability in many patients with MSA, and in our clinical series postural instability had developed in $93 \%$ of patients. ${ }^{13}$ However, relatively few patients with MSA presented with falls in the first year, whereas this is more common in PSP..$^{33}$

\section{AUTONOMIC FUNCTION}

Autonomic failure occurred in $84 \%$ of the patients with MSA, but in only $26 \%$ of the patients with PD. The range of the autonomic deficit particularly affecting orthostatic blood pressure control, micturition, and defecation is similar in both PD and MSA, although urinary incontinence and retention are more common in the second. However, deficits occur earlier in MSA and tend to also be more severe and widespread in this disorder. ${ }^{34}$ Furthermore, the anatomical basis for autonomic dysfunction is more extensive in MSA, primarily affecting preganglionic sympathetic neurons ${ }^{35}$ and the hypothalamus. ${ }^{4}$

The features that were identified as useful parts of our model to discriminate between MSA and PD are by themselves entirely non-specific. Poor levodopa response can occur in many situations, such as associated cerebrovascular disease or other atypical parkinsonian syndromes. Preserved cognition and lack of psychiatric side effects are not restricted to patients with MSA, as most patients with PD $(60 \%-80 \%)$ will also remain cognitively intact. ${ }^{29}$ Disturbed axial function is also an early hallmark of PSP, ${ }^{36}$ and also invariably accompanies end stage PD. Dysarthria, dysphagia, and falls are therefore by no means specific to MSA. Of the many different autonomic disturbances most also occur in PD and some also in PSP or other neurodegenerative disorders. However, patients with MSA are particularly prone to have severe early autonomic failure including presyncopal states, syncope, urinary incontinence or retention, impotence, and sweating disturbance. ${ }^{37}$

\section{Conclusion}

Our results suggest that improving diagnostic accuracy of MSA requires pattern recognition rather than defining unique pathognomonic symptoms or signs. In our data set matched against the gold standard of neuropathological confirmation the combination of poor or absent levodopa response, axial disturbance (dysarthria, dysphagia, dysequilibrium with falls), and intact cognition including lack of antiparkinsonian medication induced psychotoxicity yielded optimum predictive power. However, this proposed feature combination is based on follow up until death, and therefore of limited utility in early differential diagnosis. Repeat analysis restricted to early disease $(<5$ years) identified a weaker predictive model including autonomic dysfunction, poor initial levodopa response, initial rigidity, and early fluctuations. As many of the atypical parkinsonian syndromes that are seen in specialised PD clinics are due to MSA, our findings suggest that this combination could be a useful aid for "bedside" differential diagnosis. However, the utility of these features in distinguishing between MSA and PSP has not been tested, and would probably be considerably less.

These scoring systems need to be validated against different datasets. Similarly new diagnostic methods, such as neuroimaging, should be compared against simple clinical diagnosis. New incident cohorts of patients with movement disorders should be set up, so high quality prospective diagnostic data can be collected and linked to postmortem validation. Ideally this should be a multicentre collaborative venture to ensure large numbers of cases of MSA.

1 Quinn N. Multiple system atrophy: the nature of the beast. $\mathcal{F}$ Neurol Neurosurg Psychiatry 1989:52(suppl):78-89. 2 Dejerine J, Thomas A. L'atrophie olivo-ponto-cérébelleuse.

3 Adams RD, van Bogaert L, van der Eecken. DégénéresAdams RD, van Bogaert L, van der Eecken. Dégénéres-
cences nigro-striées et cérébello-nigro-striées. Psychiatria et Nences nigro-striées et cérébello-nigro-

4 Shy GM, Drager GA. A neurological syndrome associated with orthostatic hypotension. Arch Neurol 1960;2:511-27.

5 Graham JG, Oppenheimer DR. Orthostatic hypotension and nicotine sensitivity in a case of multiple system atrophy. $\mathcal{F}$ Neurol Neurosurg Psychiatry 1969;32:28-34

6 Papp MI, Kahn JE, Lantos PL. Glial cytoplasmic inclusions in the CNS in patients with multiple system atrophy (striatonigral degeneration, olivopontocerebellar atrophy and Shy-Drager syndrome). $\mathcal{F}$ Neurol Sci 1989;94:79-100.

7 Papp M, Lantos P. Accumulation of tubular structures in oligodendroglial and neuronal cells as the basic alteration in multiple system atrophy. F Neurol Sci 1992;107:172-82

8 Papp MI, Lantos PL. The distribution of oligodendroglial inclusions in multiple system atrophy and its relevance to inclusions in multiple system atrophy and its rele
clinical symptomatology. Brain 1994;117:235-43.

9 Wenning GK, Ben-Shlomo Y, Magalhães M, et al. Wenning GK, Ben-Shlomo Y, Magalhães M, et al.
Clinicopathological study of 35 cases of multiple system atrophy. F Neurol Neurosurg Psychiatry 1995;58:160-6.

10 Litvan I, Goetz CG, Jankovic J, et al. What is the accuracy of the clinical diagnosis of multiple system atrophy? A clinicopathologic study. Arch Neurol 1997;54:937-44.

1 Fearnley JM, Lees AJ. Striatonigral degeneration. A clinicopathological study. Brain 1990;113:1823-42.

12 Quinn NP, Marsden CD. The motor disorder of multiple system atrophy. I Neurol Neurosurg Psychiatry 1993;56: $1239-42$.

13 Wenning GK, Ben-Shlomo Y, Magalhães M, et al. The clinical features and natural history of multiple system atrophy. An analysis of 100 cases. Brain 1994;117:835-45.

14 Quinn N. Parkinsonism: recognition and differential diagnosis. $B M \mathcal{A} 1995 ; 310: 447-52$

15 Ben-Shlomo Y, Wenning GK, Tison F, et al. Survival of patients with pathologically proven multiple system atrophy: a meta-analysis. Neurology 1997;48:384-93.

16 Albanese A, Colosimo C, Bentivoglio AR, et al. Multiple system atrophy presenting as parkinsonism: clinical features and diagnostic criteria. $\mathcal{F}$ Neurol Neurosurg Psychiatry 1995;59:144-51.

17 Colosimo C, Albanese A, Hughes AJ, et al. Some specific clinical features differentiate multiple system atrophy (striatonigral variety) from Parkinson's disease. Arch Neurol 1995;52:294-8

18 Hughes AJ, Daniel SE, Kilford L, et al. Accuracy of clinical diagnosis of idiopathic Parkinson's disease: a clinicopathological study of 100 cases. I Neurol Neurosurg Psychiatry 1992a;55:181-4.

19 Hughes AJ, Ben Shlomo Y, Daniel SE, et al. What features improve the accuracy of clinical diagnosis in Parkinson's disease: a clinicopathologic study. Neurology 1992;42: 
20 Hughes AJ, Colosimo C, Kleedorfer B, et al. The dopaminergic response in multiple system atrophy. 7 Neurol Neuroergic response in multiple system
surg Psychiatry 1992c;55:1009-13.

21 Hughes AJ, Daniel SE, Blankson S, et al. A clinicopathologic study of 100 cases of Parkinson's disease. Arch Neurol 1993; 50: $140-8$.

22 Wenning GK, Tison F, Ben Shlomo Y, et al. Multiple system atrophy: a review of 203 pathologically proven cases. Mov Disord 1997;12:133-47.

23 Gray F, Vincent D, Hauw JJ. Quantitative study of lateral horn cells in 15 cases of multiple system atrophy. Acta Neuropathol 1988;75:513-18.

24 Quinn N. Multiple system atrophy. In: Marsden CD, Fahn S, eds. Movement disorders 3. London: ButterworthHeinemann, 1994:262-81.

25 Wenning GK, Quinn N, Magalhães M, et al. Minimal change multiple system atrophy. Mov Disord 1994;9:161-6.

26 Daniel SE. The neuropathology and neurochemistry of multiple system atrophy. In: Mathias C, Bannister R, eds. Autonomic failure. A textbook of clinical disorders of the autonomic nervous system, 3rd ed. Oxford: Oxford Univer-
sity Press, 1999:564-85.

sity Press, 1990.564

27 Mark MH, Sage JI, Dickson DW, et al. Levodopanonresponsive Lewy body parkinsonism: clinicopathologic study of two cases. Neurology 1992;42:1323-7.

28 Wagner ML, Fedak MN, Sage JI, et al. Complications of disease and therapy: a comparison of younger and older patients with Parkinson's disease. Ann Clin Lab Sci 1996;26:389-95.

29 Mayeux R, Danaro J, Hemenegildo N, et al. A populationbased investigation of Parkinson's disease with and without dementia. Relationship to age and gender. Arch Neurol 1992;49:492-7.
30 McKeith IG, Galasko D, Kosaka K, et al. Consensus guidelines for the clinical and pathological diagnosis of dementia with Lewy bodies (DLB): report of the consortium on DLB international workshop. Neurology 1996;47:111324

31 Spokes EGS, Bannister R, Oppenheimer DR. Multiple system atrophy with autonomic failure. Clinical, histological and neurochemical observations in four cases. 7 Neurol Sci 1979;43:59-82.

32 Kluin KJ, Gilman S, Lohman M, et al. Characteristics of the dysarthria of multiple system atrophy. Arch Neurol 1996;53: 545-8.

33 Litvan I, Mangone CA, McKee A, et al. Natural history of progressive supranuclear palsy (Steele-RichardsonOlszewski syndrome) and clinical predictors of survival: a clinicopathological study. I Neurol Neurosurg Psychiatry 1996;60:615-20.

34 Magalhães M, Wenning GK, Daniel SE, et al. Autonomic dysfunction in pathologically confirmed multiple system atrophy and idiopathic Parkinson's disease: a retrospective comparison. Acta Neurol Scand 1995;91:98-102.

35 Oppenheimer DR. Lateral horn cells in progressive autonomic failure. F Neurol Sci 1980;46:393-404.

36 Steele JC, Richardson JC, Olszewski J. Progressive supranuclear palsy. A heterogenous degeneration involving the brain stem, basal ganglia and cerebellum with vertical gaze and pseudobulbar palsy, nuchal dystonia and dementia. Arch Neurol 1964;10:333-58.

37 Polinsky RJ. Multiple system atrophy: clinical aspects, pathophysiology and treatment. In: Jankovic J, ed. Neurologic clinics. Vol 2. Philadelphia: Saunders 1984:487-98. 